\title{
Peertechz
}

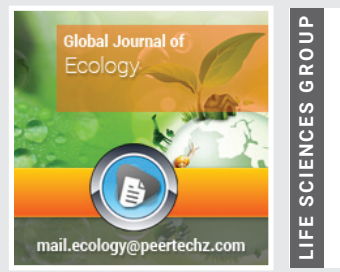

\section{Sustainable development} of urban agglomeration

\section{industrial layout based on big data and deep learning}

\section{Renzhou Gui ${ }^{1 *}$, Huilin Zheng1, Tongjie Chen ${ }^{1}$, Chuan Pang ${ }^{2}$ and Chengkun Liu $^{3}$}

${ }^{1}$ The Department of Electronic and Communication Engineering, Tongji University, Shanghai 201804, China

${ }^{2}$ School of Business, Macau University of Science and Technology, Macao, 99078, China

${ }^{3}$ Institute of Sustainable Development, Macau University of Science and Technology, Macao, 99078, China
Received: 18 January, 2021

Accepted: 02 February, 2021

Published: 03 February, 2021

*Corresponding author: Renzhou Gui, The Department of Electronic and Communication Engineering, Tongji University, Shanghai 201804, China,

E-mail: rzgui@tongji.edu.cn

https://www.peertechz.com

\begin{abstract}
Urban agglomeration is a complex movement system composed of natural environment, social economy and human on the basis of city. At present, many urban agglomerations have a large development gap between cities and lack of overall coordination, and some regions and industries still have homogeneous competition and resource misallocation. This makes the regional development of urban agglomeration unbalanced and difficult to sustainable development. By using deep learning and big data methods, this paper makes an in-depth analysis of the spatial distribution evolution of the number of enterprises and economic scale in urban agglomerations in the Yangtze River Delta region of China, analyzes the mutual influence among urban agglomerations, and discusses the methods of sustainable development.
\end{abstract}

\section{Introduction}

Sustainable development is a concept with very rich connotations. The definition of sustainable Development given by the World Commission on Environment and Development (WCED, 1987) is "the Development that meets the needs of current people without harming the needs of future generations"[1]. Urban agglomeration is a huge complex system based on the city, which is composed of natural environment, social economy, population and other factors. Low industrial level, similar structure and unreasonable layout are important factors that affect the sustainable development of urban agglomeration. At present, in China's major urban agglomerations, the output value of the tertiary industry is generally low in proportion, the technological level of the secondary industry is not high, and most of the industries are labor-intensive and capital-intensive. Internal center cities and small and medium-sized cities and urban agglomeration between the industry convergence is also quite serious, similar industrial structure, low degree of specialization, the repeated construction and the unreasonable utilization of resources, to a certain extent, affected the further coordination of regional economy, weakened the economic ties between the two cities and the improvement of the various network between cities. Therefore, it is an important way to realize the sustainable development of urban agglomeration to improve the industrial structure level, adjust the industrial structure and spatial distribution structure to optimize the allocation of environmental resources.

Traditional urban planning cannot judge the relationship between the whole and the part, let alone grasp the law of urban life and see clearly the future development of the city. Moreover, the traditional urban planning is too pure for the separation between urban functional areas. This kind of 
planning also relies on professional planners to conceive, and consumes human energy. Nowadays, the development of smart cities has become an emerging model and key goal of the world's urban development. Big data, data mining and deep learning have become important tools of smart cities. Smart city planning based on big data and deep learning can break through the constraints of data in time, space and coverage, and solve the problem of data island. The overall analysis of urban layout based on big data can evaluate the relevance between regions and between regions and the whole. Based on deep learning, the future city model can be predicted and the development direction of smart city can be rationally and scientifically determined.

Based on big data and deep learning, data collection, collation, storage, processing, visualization and analysis of urban agglomeration are carried out, conclusions are drawn and suggestions for sustainable development are put forward. Take the mature urban agglomeration in China's Yangtze River Delta as an example [2]. Firstly, Scray is used to develop a distributed framework of network acquisition for data acquisition. By analyzing website features and data location, data can be collected in a breadth-first way, and the latitude and longitude of location data can be converted by using Baidu Map Application Programming Interface (API). Next, the MongoDB database, a document-oriented database in the nonrelational database NoSQL, is used to store the data. This is a database based on distributed file storage, its query language is very powerful, can store more complex data types, has the characteristics of light weight, high efficiency and convenient transmission, high performance, easy deployment, easy to use, very convenient. The visualization of data is accomplished by Geographic Information System(GIS), which can display the data in the corresponding map in various visual ways. ArsGIS embedded in C\# programming is used to construct a special GIS application solution, which can realize the spatial visualization of data, as shown in the Figure 1 below.
Then, the convolution-deconvolution hybrid deep learning network framework is constructed to train the data. The model diagram is shown in Figure 2. The model is mainly composed of four convolutional layers and three deconvolutional layers, with a learning rate of 0.0001 and a batch size of 16 . The functions of each layer are Relu functions. It is used to learn and predict the density matrix of the $40000 \times 40000 \mathrm{~m} \wedge 2$ area with railway stations as the center before and after the time node of railway line opening, so as to obtain the development distribution density matrix of this area in the next three years. Among them, Deconvolution is a process in which convolution layers are reversed. It is widely used in image restoration and image generation.

A continuous spatiotemporal evolution prediction model [2] based on deep learning can predict the number and spatial distribution of new companies in the future, and the prediction results can reflect the possibility of the existence of enterprises in the region. Finally, from the perspective of economic correlation, the correlation coefficient matrix calculation method based on partial derivative correction can be used to analyze the spatio-temporal evolution of decades. We give the prediction of the distribution evolution of urban agglomeration enterprises using the combined loss function of MMSE-Cosin2DFFT for the loss function. For the convenience of display, only the density matrix diagram is displayed, as shown in Figure 3.

In Figure 3, the closer the color is yellow, the lower the density value (the minimum value is 0 ), the closer the color is red, the higher the density (we chose a maximum value of 10 , if the value is greater than 10 the color will be pure red). The forecast results can also reflect the likelihood of companies in the region.These results fully demonstrate that big data, artificial intelligence, deep learning and other new technologies have broad prospects in the field of smart city applications.
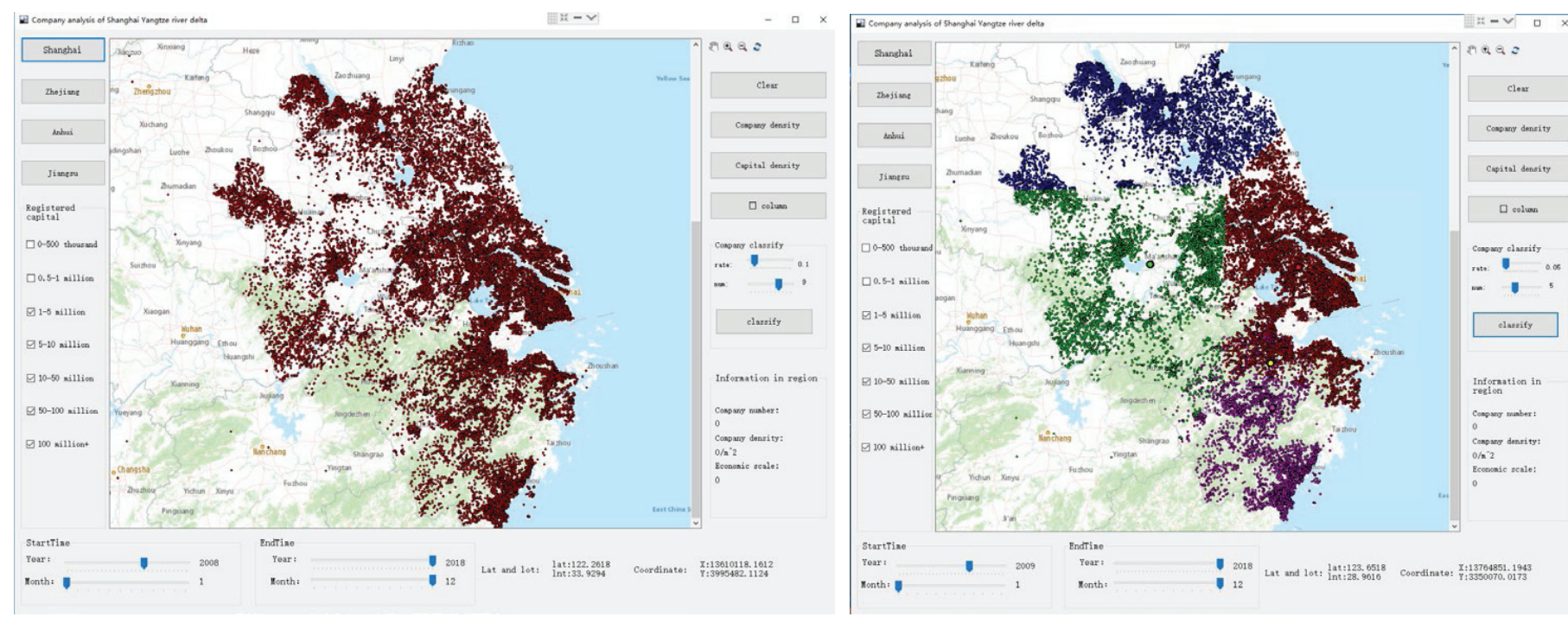

Figure 1: Data visualization interface designed in this paper. (a)Spatial distribution map of all companies collected in the 2018. (b) A result graph of clustering company data of spatial distribution using the improved $\mathrm{k}$-means algorithm (the number of clusters is chosen to be 5).

Citation: Gui R, Zheng H, Chen T, Pang C, Liu C (2021) Sustainable development of urban agglomeration industrial layout based on big data and deep learning. Glob J Ecol 6(1): 011-013. DOI: https://dx.doi.org/10.17352/gje.000039 


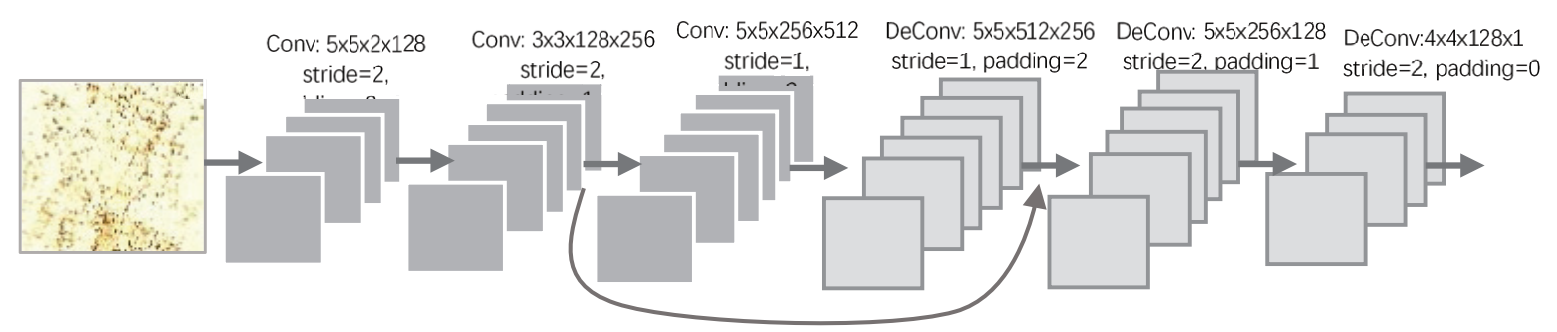

Figure 2: Deep neural network model.

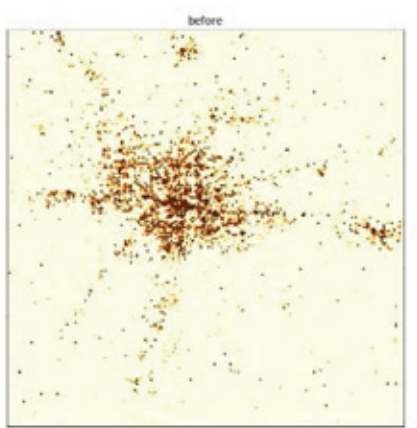

(a)

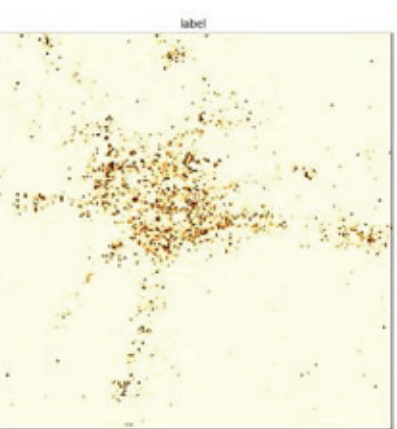

(b)

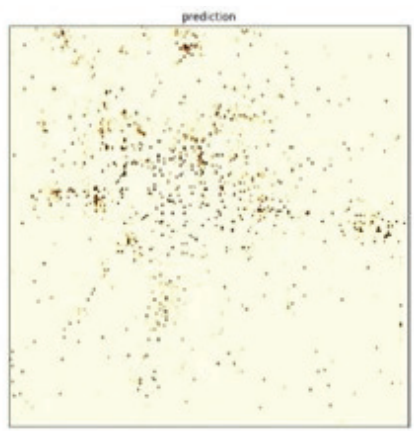

(c)

Figure 3: Shows the distribution of the number of railways before completion expressed by the test data; (b) shows the distribution of the number of newly added companies three years after completion; (c) shows the prediction results generated by the deep learning model.

\section{Conclusion}

Emerging technologies such as big data and deep learning have broad prospects in the application of smart cities, which can reveal the inherent laws of coordinated development of urban agglomerations. This idea can also be applied to the coordinated development of other smart urban agglomerations to provide conditions for the research of urban ecological sustainable development. For example, the Guangdong-Hong Kong-Macao Greater Bay Area is one of the most open and economically dynamic regions in China. The development gap between cities in the Greater Bay Area is large and there is a lack of overall coordination. Some regions and industries still have homogenized competition and resource misallocation. Therefore, unbalanced regional development and difficulty in sustainable development are one of the prominent problems in the rapid development of the Greater Bay Area that have not been well addressed. The ideas in this paper are applied to the development of urban agglomerations such as the GuangdongHong Kong-Macao Greater Bay Area, which provides a new idea for the construction of smart cities.

\section{Acknowledgement}

This work was supported by National Natural Science Foundation of China 61271351 and 41827807.

\section{References}

1. Oyeshola $D(2020)$ Despite various initiatives thus far can there be sustainable development for humanity? Glob J Ecol 5: 052-057. Link: https://bit.ly/3curdry

2. Gui $R$, Chen $T$, Nie $H$ (2020) In-depth analysis of railway and company evolution of yangtze river delta with deep learning. Complexity. Link: https://bit.ly/3oNCupz

\section{Discover a bigger Impact and Visibility of your article publication with}

\section{Peertechz Publications}

\section{Highlights}

* Signatory publisher of ORCID

* Signatory Publisher of DORA (San Francisco Declaration on Research Assessment)

- Articles archived in worlds' renowned service providers such as Portico, CNKI, AGRIS, TDNet, Base (Bielefeld University Library), CrossRef, Scilit, J-Gate etc.

- Journals indexed in ICMJE, SHERPA/ROMEO, Google Scholar etc.

- OAI-PMH (Open Archives Initiative Protocol for Metadata Harvesting)

* Dedicated Editorial Board for every journa

* Accurate and rapid peer-review process

* Increased citations of published articles through promotions

* Reduced timeline for article publication

Submit your articles and experience a new surge in publication services (https://www.peertechz.com/submission).

Peertechz journals wishes everlasting success in your every endeavours.

Copyright: ( ) 2021 Gui R, et al. This is an open-access article distributed under the terms of the Creative Commons Attribution License, which permits unrestricted use distribution, and reproduction in any medium, provided the original author and source are credited. 